Щ्)FRANÇAISE

$\supset$ DE

핌AGGGIE

\section{Revue française de pédagogie}

Recherches en éducation

$198 \mid 2017$

Regards croisés sur le baccalauréat professionnel

\title{
Les bacheliers professionnels face à Admission Post-Bac (APB) : « logique commune » versus « logique formelle » de l'orientation
}

Vocational baccalaureate holders facing Admission Post-Bac (APB): "public logic" versus "formal logic" of orientation

Claire Lemêtre et Sophie Orange

\section{OpenEdition}

Journals

\section{Édition électronique}

URL : https://journals.openedition.org/rfp/5264

DOI : $10.4000 /$ rfp.5264

ISSN : 2105-2913

\section{Éditeur}

ENS Éditions

Édition imprimée

Date de publication : 31 mars 2017

Pagination : 49-60

ISSN : 0556-7807

Référence électronique

Claire Lemêtre et Sophie Orange, « Les bacheliers professionnels face à Admission Post-Bac (APB) «logique commune » versus « logique formelle » de l'orientation », Revue française de pédagogie [En ligne], 198 | 2017, mis en ligne le 31 mars 2020, consulté le 08 janvier 2022. URL : http:// journals.openedition.org/rfp/5264; DOI : https://doi.org/10.4000/rfp.5264 


\title{
Les bacheliers professionnels
} face à Admission Post-Bac (APB) : "logique commune » versus «logique formelle » de l'orientation

\author{
Claire Lemêtre \\ Sophie Orange
}

\begin{abstract}
L'article montre que les difficultés d'usage des applications de gestion des affectations dans l'enseignement supérieur (APB hier, aujourd'hui Parcoursup), éprouvées par les élèves de terminale professionnelle et, plus largement, par les élèves des classes populaires, tiennent à un décalage entre deux façons de voir et de dire l'avenir scolaire et professionnel, chacune reposant sur une logique propre. L'application APB, loin de constituer un support neutre et transparent des choix des élèves, impose un langage singulier de ces choix (ce que nous avons appelé la «logique formelle» de l'orientation, en nous inspirant de Basil Bernstein) qui va favoriser ceux qui parlent la même langue (les bacheliers d'origine supérieure principalement) et qui va tenir à distance voire disqualifier les pratiques et les représentations de ceux qui arborent une autre logique, que nous avons appelée la «logique commune» de l'orientation.
\end{abstract}

Mots-clés (TESE) : choix des études, orientation, enseignement supérieur, formation et enseignements professionnels, situation sociale

\section{Introduction}

Les bacheliers professionnels, «tard-venus »' à la poursuite d'études et principalement issus des classes

1 On emprunte cette expression à Charles Suaud (1989). Elle implique de penser les pratiques d'orientation des bacheliers professionnels de façon relationnelle (en lien avec les pratiques des populaires (Palheta, 2012), disposent de peu de ressources scolaires et sociales pour appréhender le fonctionnement de l'enseignement supérieur comme de l'outil Admission Post-Bac (APB) qui régissait jusqu'à

autres bacheliers) mais aussi en les replaçant dans la perspective historique de leur accès à l'enseignement supérieur, relativement, là encore, aux autres bacheliers. 
récemment son accès ${ }^{2}$. En effet, leurs parents n'ont le plus souvent pas effectué d'études supérieures et les dispositifs d'aide à l'orientation ne s'adressent encore que peu à ces «nouveaux étudiants» (Erlich, 1998) en puissance.

Les témoignages ne manquent pas des problèmes rencontrés par un certain nombre d'élèves à se saisir de l'outil APB pour exprimer leurs vœux: embarras pour s'y retrouver parmi l'offre pléthorique de formations ou encore incapacité à ordonner de façon pertinente leurs candidatures (Truong, 2013). Ces maladresses ont pu être interprétées comme le résultat d'un manque d'informations à propos des filières possibles (Pistolesi, 2015) ou d'une mauvaise maîtrise technique de l'outil (Henriet \& Pietryk, 2012). Des travaux ont bien analysé la complexité du fonctionnement même de la procédure et les inégalités induites par l'algorithme de décisions, notamment concernant les consignes liées aux licences dites «en tension » et aux règles du tirage au sort (Frouillou, 2016).

Si ces difficultés sont bien réelles, le constat d'usages et d'appropriations différenciés d'APB, parfois problématiques du point de vue de leurs auteurs comme de l'institution scolaire, ne saurait selon nous $s^{\prime} y$ réduire. Il implique de réfléchir plus largement à la façon spécifique imposée par APB de « dire» ses projets scolaires et professionnels, et à sa distance plus ou moins grande avec les façons de faire des élèves, selon leurs appartenances sociales et scolaires. L'application APB fonctionne en effet comme un langage doté d'une grammaire qui conditionne des représentations de la poursuite d'études et des pratiques d'orientation. En bref, à l'instar de tout logiciel informatique d'administration et de gestion, il véhicule une certaine vision du monde, ici de l'enseignement supérieur (Eyraud, 2013).

Dès lors, il s'agit de comprendre les difficultés et les erreurs des bacheliers face à cette application non comme l'indice d'une désorientation ou de lacunes, mais plutôt comme l'expression d'un décalage entre leurs catégories de pensée et de pratique des parcours scolaires et celles prescrites par l'application. Aussi, pour mieux comprendre les désajustements d'un certain nombre d'élèves face à la démarche APB, il convient de

2 Si la loi sur l'Orientation et la réussite des étudiants promulguée au début de l'année 2018 a fait disparaître l'interface APB et I'a remplacée par l'outil Parcoursup, les éléments d'analyse développés dans cet article demeurent pleinement opérants dans la mesure où les principes qui régissent le fonctionnement de Parcoursup ainsi que son fondement idéologique demeurent chercher à reconstruire leur rationalité ou leur logique propre dans l'élaboration de leurs choix scolaires et de la comparer avec la logique institutionnelle imposée par APB.

En nous appuyant sur le cadre d'analyse proposé par Basil Bernstein à propos des différences de pratiques langagières selon les classes sociales (Bernstein, 1975), nous proposons un modèle explicatif formalisant deux logiques différentes de l'orientation scolaire post-baccalauréat, chacune ayant sa cohérence. En effet, tout comme il oppose le «langage commun » des classes populaires au «langage formel» des classes supérieures, nous suggérons de distinguer une «logique commune » de l'orientation scolaire caractéristique des classes populaires, qui vient notamment s'incarner dans les pratiques de choix et de parcours des bacheliers professionnels, et qui s'oppose à une «logique formelle» de l'orientation scolaire propre aux élèves des catégories sociales supérieures et aux bacheliers généraux. Quand la première se trouve en décalage voire en contradiction avec le langage APB, la seconde apparaît quant à elle en affinité avec le cadre et les principes de l'application.

Cet article s'appuie sur une enquête menée durant I'année scolaire 2014-2015 dans les académies de Nantes et de Créteil $^{3}$, et qui a reposé sur l'administration de 6326 questionnaires auprès d'élèves de terminales générales, technologiques et professionnelles ainsi que sur la réalisation d'observations (cours, conseils de classe, réunions d'information, salons d'orientation, etc.) et d'entretiens $(n=30)$ auprès d'élèves de terminale. Cette enquête de terrain a été complétée par l'analyse secondaire du fichier exhaustif des candidatures APB de l'académie de Nantes pour l'année 2013 et par le recueil de données statistiques auprès de certains établissements enquêtés.

Nous préciserons dans un premier temps la définition de ce que nous proposons d'appeler la «logique formelle» de l'orientation, en détaillant les attendus implicites de l'application APB. Nous préciserons dans un second temps la «logique commune» de l'orientation, caractéristique des bacheliers professionnels face à la poursuite d'études, et nous expliciterons les formes de désajustement ou de distance qu'elle induit au regard de la norme d'APB.

3 Cette enquête a été financée par le conseil régional des Pays de la Loire et par l'université Paris 8. 


\section{$L a$ « logique formelle » d'APB}

Le portail en ligne Admission Post-Bac (APB) et son mode opératoire portent un nouveau modèle de gestion de l'orientation scolaire, engagé par une série de rapports ministériels rédigés au cours des années 2000 (Hénoque \& Legrand, 2004; Tharin, 2005; Lunel, 2007; Reiss, 2007). La procédure APB, expérimentée dans l'académie de Nantes en 2006 puis étendue progressivement aux autres académies à partir de 2008, s'inscrit ainsi dans une logique de rationalisation de l'orientation (Henriet \& Pietryk, 2012), visant tout à la fois à simplifier la démarche pour l'élève et à optimiser le processus d'affectation dans l'enseignement supérieur'. En cela, cette procédure tend à concilier deux principes relativement contradictoires de l'orientation scolaire que sont le développement du projet individuel de l'élève d'une part et la gestion des flux de bacheliers d'autre part (Berthelot, 1993).

C'est également un principe de justice sociale qui est avancé pour défendre cette nouvelle gestion de I'orientation scolaire post-bac. Le portail, en fournissant des informations sur l'ensemble des filières et en réunissant en une seule procédure l'accès aux diverses formations ${ }^{5}$, propose à tous les élèves une cartographie unique et partagée de l'enseignement supérieur. En construisant un marché commun de projection, les inégalités liées aux délits d'initiés de ceux dont les ressources sociales leur rendent familiers l'enseignement supérieur et ses rouages semblent ainsi réduites. Par

4 En cela, la logique d'APB s'appuie sur une critique de la lenteur bureaucratique de l'ancien système, en plaçant au centre l'usager, dont les délais d'attente doivent être diminués. En fondant sa justification sur le confort des usagers par la simplification des démarches (Hetzel, 2006), cette nouvelle gestion de l'orientation s'inscrit dans la mouvance d'autres réformes inspirées du nouveau management public, opérées ces dernières années à l'hôpital (Belorgey, 2010), à la Poste (Vezinat, 2012) ou dans l'enseignement supérieur et la recherche (Eyraud, 2013). La conception sur laquelle s'appuie ce nouveau traitement des aspirations scolaires, à savoir le présupposé d'un élève rationnel, capable d'auto-détermination et en mesure de construire un projet d'avenir par lui-même, n'est cependant pas nouvelle. Elle est déjà présente dans la pratique de I'orientation qui se met en place à la fin des années 1970 (Huteau \& Lautrey, 1979), et qui vise à «rendre le jeune plus autonome et plus responsable dans ses conduites d'orientation » (Danvers, 1988, p. 14). La nouveauté tient dans la traduction informatique spécifique de cette conception dans l'outil centralisé qu'est APB et par son application à la majeure partie des formations de l'enseignement supérieur.

5 Si la procédure est unique en théorie, en pratique il demeure de nombreuses formations dont l'accès n'est pas pris en charge pas I'application APB. C'est le cas de nombreuses écoles supérieures ou encore de I'IEP de Paris. ailleurs, la procédure, en permettant aux élèves de multiplier leurs vœux, vise à «élever leur niveau d'ambition» ainsi qu'à leur faire "affiner leur choix» au plus près de leur projet (Hetzel, 2006). Les classements opérés par les candidats ne sont pas connus des établissements de formation demandés : I'interface APB tient confidentiel le rang des vœux ${ }^{6}$. L'automatisation permet donc d'assurer «un traitement équitable » entre les demandeurs, «égalité des chances » garantie également par la charte signée à la fois par les étudiants et les établissements scolaires, dans une véritable perspective de démarche-qualité.

Au final, c'est la neutralité mathématique (Belorgey, 2010) qui semble régir la distribution et les affectations des élèves, en dehors de toute intervention de la subjectivité humaine. Les difficultés ou problèmes rencontrés par les usagers sont dès lors le plus souvent renvoyés à des considérations d'ordre purement technique liées à une mauvaise maîtrise de l'outil, comme le rapportent Alain Henriet (Inspecteur général de l'Éducation nationale [IGEN], doyen du groupe Économie et gestion) et Gilbert Pietrick (IGEN, physique), chargés par Laurent Wauquiez, ministre de l'Enseignement supérieur et de la Recherche, d'une mission d'évaluation du dispositif APB : «la plupart de ceux qui rencontrent des difficultés reconnaissent n'avoir pas pris conscience suffisamment tôt des enjeux du dispositif ou n'avoir pas suffisamment approfondi la lecture des guides d'accompagnement ou négligé les messages d'alerte» (Henriet \& Pietryk, 2012). Les solutions proposées s'expriment ainsi généralement sur le registre de l'assistance technique, au moyen d'applications Smartphones, de foires aux questions sur internet, de salons ou de stands au sein des salons d'orientation spécialement dédiés au fonctionnement de la procédure, ou encore par l'amélioration des fonctionnalités de l'outil ${ }^{8}$.

Mais en faisant oublier les « lois sociales de construction » de son langage (Bourdieu \& Boltanski, 1975), le dispositif APB contribue à nier non seulement l'arbitraire de son fonctionnement, mais aussi les affinités de la conception de l'orientation qu'il porte avec le

$6 \quad$ Ce principe est de plus en plus remis en cause actuellement avec l'instauration de tirages au sort dans les licences universitaires à capacités d'accueil limitées, où le rang du vœu et/ou l'académie d'origine peuvent constituer des critères de sélection.

7 Ce principe est considéré comme le «principe fondateur du dispositif » (Henriet \& Pietryk, 2012).

8 Dossier de presse APB du 8 décembre 2015. En ligne : <http:// cache.media.education.gouv.fr/file/12_-_decembre/37/9/2015_DP_ APB_510379.pdf> (consulté le 8 juillet 2016). 


\begin{tabular}{l|l}
\hline Logique formelle & Logique commune \\
\hline - Gamme assez étendue de choix possibles & - Gamme limitée de choix possibles \\
- Arrangements originaux des choix & - Arrangements prévisibles des choix \\
- Rapport abstrait aux choix & - Expérience directe aux choix \\
- Expérience personnelle unique & - Démarche collective \\
- Démarche distinctive & - Conservatisme scolaire \\
\hline
\end{tabular}

Note : cette formalisation est très largement mais aussi très librement inspirée des principes structurant le langage formel et le langage commun développés par Bernstein (1975, p.25-62), sans ignorer les débats que ces travaux ont engendrés avec Labov. L'analogie avec Bernstein se veut en effet être un outil pour expliciter un certain nombre de régularités observées dans les logiques d'orientation des bacheliers. La grille construite par Bernstein permet d'établir une typologie non normative, qui vise à rendre toute sa richesse et sa forme propre à la logique d'orientation des bacheliers issus des classes populaires, en bref à la définir en termes de différences et non en termes de manques.

mode de pensée et de projection des catégories supérieures, ce que nous proposons d'appeler la «logique formelle » de l'orientation.

La procédure APB invite les élèves de terminale à formuler, sur une séquence temporelle comprise entre janvier et mai, leurs vœux de candidature pour les formations de l'enseignement supérieur. Le déroulement de la phase d'affectation qui intervient au terme de cette séquence, une fois que les commissions de sélection des établissements d'accueil ont procédé à leurs arbitrages, consiste à proposer au candidat le vœu le plus haut de son classement auquel il a été admis, et uniquement celui-ci. Les vœux situés plus bas dans ce classement sont automatiquement supprimés et le candidat a le choix soit d'accepter définitivement la proposition qui lui est faite (oui définitif), soit de l'accepter mais d'attendre de savoir s'il peut obtenir un vœu mieux placé dans sa liste, à la faveur de désistements d'autres candidats (oui mais), soit de refuser cette proposition et d'attendre de savoir s'il peut obtenir un vœu mieux placé (non mais). Cette gestion des affectations en flux tendu implique pour le candidat d'opérer son choix avant même de connaître le résultat des admissions et conduit les élèves disposant de moins de ressources scolaires et sociales à respecter l'ordre des risques plutôt que l'ordre des préférences (Bodin \& Orange, 2013). On a en effet montré ailleurs que certains élèves positionnaient en premier vœu non pas la formation préférée ou la plus difficile d'accès, mais la formation où ils estimaient qu'ils avaient le plus de probabilités d'être acceptés (Orange, 2013).

Le fonctionnement d'APB engage donc à optimiser le nombre et l'ordre de ses vœux, de façon à « multiplier [s]es chances d'accès $»^{9}$. Cela implique d'avoir une maîtrise assez vaste de l'espace de l'enseignement supérieur, d'en connaître la hiérarchie et les rouages internes, et de pouvoir se positionner dans une logique anticipatrice (Bourdieu, 1977). Cela nécessite également d'être en mesure de penser son avenir scolaire et professionnel en raisonnant de façon abstraite sur la base des intitulés des formations, puisque l'entrée dans l'application se fait selon les types de filières (BTS, IUT, licences, etc.), avant même d'envisager des spécialités disciplinaires ou professionnelles, et enfin les localisations concrètes de ces formations. En cela, le fonctionnement de cette application se trouve en affinité avec les représentations et les pratiques d'orientation caractéristiques des classes supérieures et des bacheliers généraux ${ }^{10}$, qui procèdent à (1) une gamme assez étendue de choix possibles, (2) des arrangements originaux de ces choix, (3) un rapport abstrait aux choix. II conforte et favorise également une conception de la poursuite d'études vécue comme (4) une expérience personnelle unique et selon (5) une démarche distinctive (van Zanten, 2009) (voir tableau 1).

Afin d'incarner cette logique, nous proposons de rapporter le cas de Paula, fille d'un greffier en chef et d'une attachée de recherche clinique. Elle est en terminale ES section Abibac ${ }^{11}$ dans un lycée du centre-ville

9 Entretien avec la responsable adjointe d'un service académique d'information et d'orientation (SAIO), février 2015.

10 Tout comme les classes supérieures ont la double maîtrise du «langage formel» et du «langage commun», selon le modèle de Basil Bernstein, les élèves des classes supérieures ont la double maîtrise de la «logique formelle» et de la «logique commune» de l'orientation.

11 L'Abibac est un examen unique qui permet la délivrance simultanée du baccalauréat français et de son équivalent allemand ( $\mathrm{Abi}$ tur) depuis 1994 
de Nantes. Paula constitue en quelque sorte un idéaltype de la logique formelle. Elle se montre non seulement à l'aise pour formuler plusieurs vœux, mais aussi pour les hiérarchiser. Elle conçoit ainsi tout à fait le principe de déclinaison attendu par APB, qui consiste à établir plusieurs scénarii et à anticiper des plans $B$ : "L'idée [de départ] c'était Sciences Po, après ça été oui, I'allemand à tout prix et au fur et à mesure, ça s'est construit, donc c'était assez long. Et après, la question, parce qu'il n'y a quand même que 10 places à chaque fois, c'était... il faut un plan B et après vu tout l'investissement, tout le travail que j'ai donné, je me suis dit, il faudrait le rentabiliser en retentant après le concours... et du coup, ça a été l'idée d'une prépa, parce que je pense que j'ai les capacités pour travailler... ». Paula a en effet formulé 15 vœux dans l'application alors que le nombre de vœux moyen, toutes filières confondues est de 5 , et de 6 pour la seule série $S^{12}$. Elle fait donc partie de ces élèves usant pleinement des possibilités de choix proposées par le logiciel. "La conseillère d'orientation a préconisé au moins six vœux, après moi, j'avais plusieurs choses qui m'intéressaient et puis je ne savais pas trop, je me disais : "il vaut mieux faire"... je suis plutôt prévoyante, "il vaut toujours mieux en faire trop et après, on changera l'ordre"... qu'après, de ne pas être prise à Sciences Po et de le savoir qu'en avril et après de regretter de n'avoir que des prépas par exemple... ». Ses propos rendent compte en creux de l'ajustement entre la logique anticipatrice du logiciel et ses propres dispositions («je suis plutôt prévoyante $)^{13}$. Cette élève précise par ailleurs au cours de l'entretien qu'elle a passé beaucoup de temps, seule, sur APB («j'ai quand même épluché le site»), révélant par là même son appréhension méthodique et organisée de l'outil. Ce travail lui a permis de parfaire ses connaissances dans le domaine des formations postbac relatives à son projet de poursuite d'études : «Les descriptions des formations sur APB étaient très précises, ça m'a permis aussi de découvrir d'autres formations par APB.» Paula a réussi à se construire, à l'aide notamment du logiciel APB, sa propre cartographie de l'enseignement supérieur : une cartographie personnalisée, au plus près de son projet de poursuite d'études. Ce véritable travail topographique lui offre une parfaite

12 Source: APB 2013, académie de Nantes.

13 Philippe Gombert montre ainsi comment les parents des classes supérieures sont suffisamment familiers de la culture de l'enseignement supérieur pour engager une vision de l'éducation sur le long terme et anticiper de façon rationnelle les différentes étapes d'un parcours (Gombert, 2008). maîtrise du segment de l'enseignement supérieur dans lequel elle va évoluer d'ici quelques mois. Comme elle le précise à l'enquêtrice : « Je ne vois pas d'autres choix que j'aurais pu faire, il n'y a pas d'autres formations», révélant ainsi le caractère exhaustif de ses vœux. De plus, I'approche scolaro-centrée, c'est-à-dire par le prisme des formations plutôt que des métiers, qui préside au fonctionnement du logiciel, s'ajuste parfaitement à la manière dont Paula appréhende l'enseignement supérieur, qui ne se pense que dans la poursuite d'études, n'évoquant à aucun moment une profession visée en particulier. À la toute fin de l'entretien, lorsque Paula est interrogée sur «des envies de métiers? Tu te verrais faire quoi?», sa réponse est sans appel : «Pas du tout».

De façon dialectique, la «logique commune» de I'orientation, caractéristique des bacheliers professionnels, consiste en (1) une gamme limitée de choix possibles, (2) des arrangements prévisibles et (3) une expérience directe de ces choix. Elle s'accompagne d'une (4) démarche collective dans l'orientation et se traduit par (5) un certain conservatisme scolaire. Il s'agit à présent d'en préciser les termes et de saisir en actes les effets de désajustement et de décalage qu'elle produit dans le rapport de ces bacheliers à l'application APB.

\section{Un système restreint}

Une première caractéristique des vœux formulés par les élèves de terminale professionnelle au seuil du baccalauréat est le caractère restreint de leur nombre et de leur type ${ }^{14}$. Les candidatures à la poursuite d'études de ces bacheliers se déclinent en effet selon une gamme limitée des vœux qui s'affiche comme une première spécificité de ces publics.

Le calcul de la moyenne des vœux réalisés montre une différence nette entre les bacheliers professionnels et les bacheliers technologiques ou généraux. Alors que les élèves des voies générales affichent une moyenne de 5,3 vœux formulés, avec des pratiques assez hétérogènes (écart-type de 4,9), les élèves des voies professionnelles affichent une moyenne de 3,9 vœux formulés, avec des pratiques plus homogènes (écart-type de 3,0). Ce sont encore $14,3 \%$ des élèves de baccalauréat général et $11,6 \%$ des élèves de baccalauréat technologique qui

14 «Pour qui parle le langage commun, les choix et les arrangements personnels sont très restreints, alors que celui qui parle le langage formel peut faire des choix et des arrangements tout à fait originaux» (Bernstein, 1975, p.30). 
Tableau 2. Caractéristiques des candidatures APB en fonction de la filière de baccalauréat

\begin{tabular}{|c|c|c|c|c|c|c|}
\hline & $\begin{array}{l}\text { Nombre } \\
\text { moyen de } \\
\text { vœux }\end{array}$ & Écart-type & $\begin{array}{c}10 \text { vœux et } \\
\text { plus }\end{array}$ & $\begin{array}{c}\text { Nombre de } \\
\text { vœux } \\
\text { maximum }\end{array}$ & Un seul vœu & $\begin{array}{l}\text { Un seul type } \\
\text { de formations } \\
\text { demandé }\end{array}$ \\
\hline Générale & 5,3 & 4,9 & $14,3 \%$ & 108 & $15,0 \%$ & $47,3 \%$ \\
\hline Technologique & 5,2 & 3,8 & $11,6 \%$ & 48 & $9,8 \%$ & $49,0 \%$ \\
\hline Professionnelle & 3,9 & 3,0 & $6,4 \%$ & 35 & $20,0 \%$ & $84,8 \%$ \\
\hline Ensemble & 5,0 & 4,4 & $12,4 \%$ & 108 & $14,7 \%$ & $54,0 \%$ \\
\hline
\end{tabular}

Source : APB 2013, académie de Nantes.

Note : pour les colonnes 3, 5 et 6 , test du Khi2 significatif au seuil de $p=0,05$.

demandent 10 vœux et plus, quand ce n'est le cas que de $6,4 \%$ des élèves de baccalauréat professionnel. Le nombre de vœux maximum formulés descend avec la position dans la hiérarchie scolaire : il est de 108 vœux chez les bacheliers généraux, 48 chez les bacheliers technologiques et enfin 35 chez les bacheliers professionnels. De même, si les élèves des voies générales et technologiques présentent des paniers de vœux largement fournis, ils se distinguent également des élèves des voies professionnelles par une diversification plus importante des types de filières demandées. Les bacheliers professionnels sont à la fois ceux qui présentent le plus de candidatures uniques $(20 \%$ contre $15 \%$ pour les bacheliers généraux et $9,8 \%$ pour les bacheliers technologiques) et ceux qui ne demandent en plus forte proportion qu'un seul type de formation $(84,8 \%$ contre $47,3 \%$ des bacheliers généraux et $49 \%$ des bacheliers technologiques) (voir tableau 2).

Cette gamme limitée de choix opérés par les élèves de terminale professionnelle est à mettre en lien avec le caractère lui-même limité de l'offre de formations qui est proposée à ces élèves. En effet, ces derniers se voient davantage que les élèves des autres filières circonscrits par les agents de l'orientation scolaire (conseiller d'orientation psychologue, enseignants, chefs d'établissements) à une seule fraction de l'enseignement supérieur, en l'occurrence les BTS (Orange, 2010). On comprend alors que les modalités d'agencement des vœux soient beaucoup plus limitées chez ces élèves que pour les élèves des filières générales mais aussi technologiques, du fait de ces prescriptions restrictives.

La possibilité offerte par APB de multiplier presque à l'envi les vœux ${ }^{15}$, donnant un sentiment de liberté et

15 Les candidats étaient théoriquement limités à 36 vœux jusqu'en 2016 ; ils sont contraints désormais à 24 vœux maximum. un pouvoir important au candidat, vient également se heurter à des formes et des logiques de pensée fortement distinctes. Le fonctionnement de la procédure APB invite, on l'a dit, à formuler plusieurs scénarii d'orientation, c'est-à-dire à décliner, pour un projet professionnel défini, différentes voies de formation. Mais cette compétence s'avère en réalité inégalement partagée et nombreux sont les élèves à se trouver démunis face à la double injonction - qu'ils ressentent parfois comme contradictoire - à devoir à la fois bâtir un projet professionnel solide mais aussi à multiplier les candidatures dans l'enseignement supérieur ${ }^{16}$.

Anthony est en terminale Électrotechnique énergie équipements communicants (ELEEC) à Angers. Son père, originaire de l'île Maurice, est retraité, après avoir été concierge dans un château angevin puis magasinier dans une enseigne de bricolage. Sa mère, préparatrice de commandes, a été licenciée pour des raisons économiques. Il souhaite entrer en licence Maintenance hôtelière hospitalière et immobilière à l'université d'Angers. S'il se plie à la consigne qui consiste à multiplier les vœux dans $\mathrm{APB}$, c'est sans en comprendre la logique. Anthony déploie ainsi deux autres vœux à la suite de son projet d'orientation, et ce davantage par docilité à l'égard des injonctions scolaires que par véritable stratégie. En effet, s'il postule en premier vœu à une licence non sélective, où il est sûr d'être accepté, il ajoute, en second vœu, le BTS Conception et réalisation de systèmes automatiques (CRSA) de son lycée puis un autre BTS, en vue d'arriver à 3 vœux:

16 D'autant plus que les élèves de baccalauréat professionnel sont, à l'instar des élèves orientés dans les filières les plus dévalorisées du système scolaire, ceux sur lesquels pèse le plus fortement cette «injonction au projet», ces bacheliers étant régulièrement sommés de justifier leur souhait d'entrer dans l'enseignement supérieur, quand celui-ci est considéré comme normal voire naturel chez les bacheliers généraux (Beaud \& Pialoux, 2001). 
Tableau 3. Situation face à APB des élèves de terminale du lycée polyvalent Catherine de Parthenay* (Vendée) (en \%)

\begin{tabular}{l|c|c|c|c}
\hline & Non inscrit & $\begin{array}{c}\text { Pas de vœux } \\
\text { exprimés }\end{array}$ & $\begin{array}{c}\text { Vœux exprimés dans } \\
\text { APB }\end{array}$ & Total (effectifs) \\
\hline Générale & 1,0 & 0 & 99,0 & $100 \%(101)$ \\
\hline Technologique & 10,5 & 0 & 89,5 & $100 \%(19)$ \\
\hline Professionnelle & 30,5 & 11,0 & 58,5 & $100 \%(82)$ \\
\hline Ensemble & 13,9 & 4,5 & 81,6 & $100 \%(202)$ \\
\hline
\end{tabular}

Source : données de l'établissement, 2015.

Note : : le nom de l'établissement a été modifié.

Anthony : Et en $3^{\text {e }}$ choix, j'avais pris BTS Électricité, $c^{\prime}$ est juste pour avoir un $3^{e}$ choix... [léger rire].

Enquêtrice : Parce que, tu dis?

Anthony : Non, c'est comme ça... j'ai pris parce qu'il

fallait mettre un $3^{\mathrm{e}}$ vœu...

Enquêtrice : Fallait, c'est-à-dire?

Anthony: On m'a dit faut mieux mettre au moins des choix juste pour être sûr...

Enquêtrice : Qui est-ce qui t'a dit?

Anthony: C'est mes profs, juste pour être sûr, c'est un plan de sauvetage, quoi...

Enquêtrice : Donc tu as fait trois vœux, et ce BTS, il est où?

Anthony : Je crois qu'il est à Saint-Sylvain d'Anjou, je ne suis pas sûr...

Enquêtrice : [...] Et c'est en ville, à Angers ?

Anthony: Non, je ne sais pas du tout... j'ai pris le plus proche en fait, je ne me suis pas pris la tête.

Lorsqu'il explique son mode opératoire, il apparaît que son classement relève davantage d'une soumission à une prescription qu'à un ordre réfléchi et optimal eu égard au fonctionnement d'APB. En effet, son premier vœu étant assuré d'être satisfait, le recours à des vœux "de sauvetage», comme il dit, n'a pas lieu d'être; mais en outre, le choix d'un «plan de sauvetage» dans une filière sélective et un lycée inconnu ne constitue pas une sécurité. Les candidatures d'Anthony traduisent juste qu'il a compris qu'il fallait multiplier les vœux mais n'a pas compris le principe d'ordre des risques qui doit régir le classement, en ordonnant les formations depuis la préférée jusqu'à la moins souhaitée, mais aussi en ordonnant les formations préférées selon leur degré de difficulté d'admission décroissante.

Le fonctionnement d'APB, qui engage à formuler plusieurs vœux pour un même projet et à savoir les ordonner, apparaît ainsi parfaitement opaque pour un certain nombre d'élèves, qui appliquent la consigne mais sans la comprendre, produisant des classements non rentables ou non stratégiques du point de vue de la logique d'APB, voire parfois même contre-productifs.

Si les bacheliers professionnels se trouvent éloignés du langage APB, certains se tiennent littéralement à l'écart de la procédure. La base académique APB ne prenant en compte que les candidatures effectuées, elle ne permet pas de mesurer la part non négligeable des bacheliers professionnels qui, soit ne s'inscrivent pas dans l'application, soit n'y formulent pas de vœux. L'accès à un fichier exhaustif des situations à l'égard d'APB d'un lycée polyvalent vendéen accueillant 202 élèves de terminale rend en revanche visible toute une population d'abstentionnistes, et distingue nettement là encore les bacheliers généraux et technologiques des bacheliers professionnels. Quand la quasi-totalité des élèves de terminale générale s'expriment dans APB, seuls $18,2 \%$ des élèves de terminale Menuiserie aluminium verres (AVM) ou encore $44,4 \%$ des élèves de terminale Maintenance des équipements industriels (MEI) enregistrent des vœux (voir tableau 3).

Ce résultat est cohérent avec le taux de poursuite d'études à l'issue du baccalauréat, qui ne concerne que $35,2 \%$ des bacheliers professionnels, contre $75,5 \%$ des bacheliers technologiques et $98,5 \%$ des bacheliers généraux (MENESR, 2014). Néanmoins, il masque un nombre non négligeable de poursuites d'études qui ne trouvent pas à s'exprimer dans les contours d'APB. L'enquête par questionnaires montre ainsi que plus d'un élève de terminale professionnelle sur cinq envisagent un premier vœu «sous le baccalauréat» ou en marge de l'enseignement supérieur tel qu'il est défini par APB, c'est-à-dire à destination de formations ou de concours dont le niveau d'entrée est inférieur au niveau IV de qualification (CAP, autres baccalauréats, brevet professionnel, formations paramédicales ou sociales), de formations de la sécurité des biens et des personnes non 
répertoriées dans APB (armée, police, pompiers) ou encore de qualifications extra-scolaires comme les certifications sportives. Or ces choix ne sont pensés par les élèves qui les formulent ni comme des réorientations ni comme des formes de déclassement scolaire, mais bien comme les prolongements cohérents d'un parcours de formation qui, de fait, échappe totalement à la logique d'échelle de l'institution qu'incarne le dispositif APB (Lemêtre \& Orange, 2017).

\section{L'« expérience directe» de la poursuite d'études}

Un autre aspect de la logique commune de l'orientation, caractéristique des bacheliers professionnels, est le rapport direct à l'avenir scolaire. Cela signifie que les élèves pensent la poursuite d'études à partir de lieux familiers ou clairement identifiés, et non depuis des intitulés ou des contenus abstraits de formation. Cette approche de l'orientation, qu'on pourrait qualifier de pratique, continue d'expliquer le caractère limité des choix formulés par ces publics, qui pensent l'avenir depuis ce qu'ils connaissent et ce qu'ils ont expérimenté directement (Beaud, 2002). Lorsqu'il est interrogé sur sa connaissance de l'Université, Anthony ne pense pas à l'institution d'enseignement supérieur en général, mais considère uniquement l'université d'Angers, qu'il appelle plus volontiers par le nom de son campus "Saint-Serge». Et plutôt que de répondre sur l'organisation et le fonctionnement du cursus, il évoque son expérience directe de l'établissement :

Enquêtrice : Et sur l'Université, ce qu'on a pu te dire à ce moment-là, toi, tu connaissais l'Université?

Anthony: Ah, oui, je l'ai vue quoi [l'université], parce que c'est à côté du cinéma, du cinéma Gaumont. Oui, c'est à côté du cinéma, alors souvent, quand on va au cinéma, on passe devant. Mais je ne savais pas du tout qu'il y avait une licence dedans, je croyais que c'était plus scientifique ou... les arts.

Face à cette appréhension immédiate de l'enseignement supérieur, l'application APB oppose au contraire un rapport indirect à la poursuite d'études et impose de pouvoir raisonner à partir des appellations génériques des filières. II s'agit en effet de raisonner d'abord sur des types de filières (BTS, DUT...), avant de choisir une spécialité, pour enfin introduire, mais seulement en dernière instance, des éléments de localisation. Dans cette logique déconnectée du vécu immédiat, retrouver perdus car ne disposant pas des moyens ou des codes pour raisonner de façon abstraite sur la seule base des intitulés de formation. Cette manière de s'orienter nécessite de savoir se situer et se projeter dans la cartographie abstraite du schéma de I'ONISEP (Office national d'information sur les enseignements et les professions), là où beaucoup de ces élèves s'orientent par les lieux. L'enseignement supérieur correspond en effet pour beaucoup de lycéens à un espace localisé, associé à des lieux éprouvés par le biais d'amis ou de membres de la famille. En cela, les élèves veulent souvent moins faire « un » BTS en général ou même « un » BTS Commerce international de façon abstraite, que «le» BTS du lycée ou de tel autre établissement connu en particulier. Anthony explique ainsi : «Au début, je pensais aller à CRSA et puis quand on a présenté IMIS [Institut de Maintenance immobilière et sécurité], je trouvais ça beaucoup plus intéressant. ». L'expression «aller à CRSA», qui désigne l'orientation dans le BTS Conception et réalisation de systèmes automatiques proposé dans l'établissement où il prépare son baccalauréat, est révélatrice de ce qui est visé : la localisation plutôt que la formation. Cela se traduit dans les vœux qu'Anthony opère, où il ne décline pas les différents établissements qui proposent ce BTS mais se contente de ne mentionner que la formation connue directement («ils sont au lycée»). Ce décalage entre les catégories de pensée et d'action de la plupart des élèves de terminale professionnelle et celles de l'application APB se retrouve dans les propos d'Anthony, lorsqu'il relate sa saisie des vœux dans le logiciel et les difficultés rencontrées:

Anthony : Je voyais bien que c'était bien cadré, il faut juste suivre [la procédure]. Après, c'est chercher les poursuites d'études, ça, c'est un peu plus... il faut avoir le nom [léger rire]...

Enquêtrice : Mais toi, tu savais déjà...

Anthony : Mais le problème, c'est que c'est pas marqué IMIS! C'est Génie civil [léger rire] après, fallait trouver!

La classification de l'enseignement supérieur d'APB impose de lire et de dire la poursuite d'études depuis un cadre scolaire, alors même qu'un grand nombre des élèves de baccalauréat professionnel pensent d'abord à un métier avant même de pouvoir l'exprimer dans une formation. C'est le cas de Quentin, en terminale ELEEC dans un lycée professionnel de la métropole nantaise :

Quentin : Par exemple, pour me renseigner sur les BTS, j'allais sur... d'abord, j'allais sur les fiches Pôle Emploi, des métiers, pour voir à quoi ça correspondait 
tel BTS, et après, je me renseignais [sur le BTS] [...] Parce que ma copine, elle s'était inscrite chez Pôle Emploi et puis, elle m'avait dit : «ouais, il y a toutes les fiches des métiers dessus ».

Enquêtrice : Tu partais du métier pour...

Quentin : Oui, voilà pour savoir quel BTS il fallait prendre. [...] Mon père m'avait offert, il y a longtemps, un livre : Les 230 métiers de France. J'ai eu I'occasion de regarder [sur un ton amusé].

Enquêtrice : C'est tous les métiers qui existent?

Quentin : Voilà, tous les métiers, par exemple, dans les espaces verts, dans les usines...

Enquêtrice : Pourquoi il t'avait offert ça?

Quentin : C'était quand j'étais en $3^{\mathrm{e}}$, et que justement, j'allais me décider si j'allais en pro, en général...

Cette approche de l'avenir scolaire à rebours, depuis le marché du travail, constitue un point d'achoppement qui va tenir un certain nombre d'élèves en marge de I'application. Alexia, élève en terminale Commerce à Saint-Nazaire, reconnaît ainsi avoir été découragée par APB car, après s'être inscrite assez facilement sur l'interface, elle n'a pas trouvé, comme elle dit : «comment envoyer les lettres de motivation et les $C V$ », pour désigner les dossiers de candidature à transmettre aux établissements d'accueil. Elle transpose ainsi la même démarche et le même vocabulaire qu'elle a mis en œuvre pour trouver une entreprise en alternance, là où le fonctionnement de la procédure APB exige un dossier académique et non un « $\mathrm{CV}$ » à proprement parler. Elle préfère alors ne pas valider ses vœux, justifiant son abandon en se positionnant toujours depuis l'emploi : «Franchement je suis trop perturbée en fait, c'est ça. Déjà, cette année, c'est stressant parce que là je vois dans deux semaines je passe le bac, et j'ai l'impression qu'on n'est pas assez renseignés sur les métiers qu'il y a. Je sais qu'il y a plusieurs métiers qu'on ne connaît pas; [donc] nous on prend les métiers basiques. »

Si APB constitue un langage par lequel l'enseignement supérieur se donne à voir de manière particulière et qui va se trouver plus ou moins en affinité avec les logiques d'orientation des élèves, il va également constituer un prisme à travers lequel les aspirations des bacheliers vont se réfracter (changer de direction, subir une inflexion) sous l'effet des prescriptions émanant des divers agents de l'orientation scolaire. Au-delà du portail numérique, la procédure APB constitue en effet une véritable matrice de pensée à travers laquelle les aspirations scolaires des élèves vont être normalisées. Cette action de l'institution scolaire va prendre la forme d'une disqualification des candidatures qui ne s'inscrivent pas dans le format APB, pour ensuite se traduire par la proposition voire l'imposition de projections qui se disent quant à elles dans la terminologie d'APB, aussi bien en termes de contenus (types de vœux) que de logiques de classement (imposition de stratégies) ${ }^{17}$. Dans un lycée polyvalent de Seine-et-Marne, une élève de terminale Commerce éprouve par exemple toutes les difficultés à savoir comment exprimer son projet sous forme de vœu scolaire, comme cela est demandé dans le questionnaire soumis :

Kimberley : Moi je veux faire des concours, donc je mets quoi?

Enquêtrice : Des concours pour faire quoi?

Kimberley : Des concours pour être responsable.

Enquêtrice : Vous voulez passer des concours pour après faire des études?

Kimberley: Non, c'est juste des concours.

L'enseignante présente pendant l'administration du questionnaire intervient alors pour expliquer à l'élève que cela n'existe pas des concours pour être responsable, et qu'il faut faire un BTS. L'élève semble déçue : elle fait actuellement un stage chez Maxi Toys et souhaitait profiter d'un «concours» pour accroître ses chances d'être embauchée. Il apparaît bien en cela que le rapport à la qualification s'exprime pour Kimberley depuis une situation concrète et localisée de travail, mais ne se perçoit pas selon une approche scolaire et déconnectée de l'expérience vécue. Par son discours, l'enseignante va chercher à traduire en bonne et due forme le projet de Kimberley. Soit elle continue ses études et obtient une qualification plus élevée, soit, si elle a une opportunité d'embauche, elle peut la saisir et espérer grimper les échelons au sein de l'entreprise. Kimberley finit par corriger son formulaire en rayant la mention «faire des concours» qu'elle avait écrite initialement et en la remplaçant par «faire des formations». Elle surenchérit néanmoins auprès de son

17 La requalification des projets des élèves s'opère également dans le cadre du "travail d'orientation» assuré par les conseillers d'orientation, les professeurs principaux et plus largement les équipes pédagogiques et administratives des lycées. Durant notre enquête, nous avons ainsi pu observer des conseils de classe du second semestre du lycée polyvalent Catherine de Parthenay où les élèves étaient évalués sur leur capacité à formuler un appariement rationnel de vœux. S'opposent ainsi l'élève qui propose un ensemble "très cohérent»; l'élève qui "a l'intelligence de mettre les deux : alternance et voie scolaire»; l'élève qui demande une formation qui «lui va comme un gant» à l'autre à qui on dit : «ça t'irait bien DCG [Diplôme de Comptabilité et de Gestion] »; I'autre à laquelle on concède qu'«elle a toujours parlé de l'hôtellerie-restauration », tout en suggérant que «BTS Assistant de manager, c'est ce qui lui irait le mieux». Il est apparu que les processus de disqualification et de retraduction des projets étaient fortement corrélés à l'origine sociale de ceux qui les portaient. 
enseignante : "Mais je ne peux pas faire d'autres concours pour avoir des acquisitions? » Ce qui apparaît dans cet exemple mais également dans l'observation des conseils de classe du second semestre de l'année de terminale d'un lycée polyvalent, c'est le travail de conversion opéré par les agents de l'orientation scolaire et qui consiste à exprimer les projets des élèves dans le langage d'APB voire même à leur imposer des projets APB de toute pièce lorsque les projets ne peuvent faire l'objet d'une traduction.

Les rapports à l'avenir qui ne peuvent s'exprimer dans la taxinomie d'APB sont de fait considérés comme nuls et non avenus (Willis, 1978; Rochex, 1995). Le logiciel de gestion des vœux endosse alors une réelle dimension performative en ne faisant exister que les projets qui peuvent s'y traduire et s'y exprimer. L'action normalisatrice d'APB agit donc sur et par les prescripteurs d'orientation scolaire qui ne sont dès lors disposés à ne reconnaître qu'une seule façon de classer l'avenir. L'expression des vœux dans l'application APB n'est donc pas seulement une opération de traduction, mais aussi, pour ceux qui sont les plus éloignés de son mode de pensée, une véritable opération de conversion des projets.

\section{Mobilisation collective et conformisme scolaire}

La logique commune de l'orientation, déployée par les élèves de baccalauréat professionnel, se caractérise également par un rapport collectif à la poursuite d'études, contrairement aux élèves des catégories supérieures et des classes générales, où l'orientation procède d'une démarche individuelle et stratégique au sein d'APB. En effet, les élèves de terminale professionnelle, qui ne peuvent s'appuyer sur l'expérience personnelle de leurs parents, sont contraints de chercher dans le groupe de pairs un appui alternatif. Cet «impératif de la camaraderie» (Hoggart, [1957] 1970, p. 125) s'incarne en premier lieu dans des pratiques collectives de prise d'information. Nombreux sont les élèves de terminale professionnelle rencontrés à avoir indiqué se rendre dans les salons d'orientation ou aux portes ouvertes d'établissements à plusieurs, avec leur groupe d'amis.

Àl'inverse, ils sont beaucoup moins nombreux que leurs homologues de terminale générale ou technologique à avoir bénéficié des dispositifs individualisés d'aide à l'orientation : seulement 15,9\% d'entre eux conseiller d'orientation psychologue contre 35,5\% des élèves de terminale générale et $35,6 \%$ des élèves de terminale technologique. Ce rapport collectif à la poursuite d'études se traduit également par des choix opérés en groupe. Fabien est élève de terminale Pilote de ligne de production dans le même lycée qu'Anthony. II est le premier dans sa famille à pouvoir prétendre poursuivre des études dans l'enseignement supérieur. Son père est gardien de déchetterie après s'être formé au métier de couvreur. Une blessure au bras l'a contraint à changer de profession. Sa mère travaille dans un supermarché. L'objectif de Fabien concernant sa poursuite d'études est dès lors de fuir le monde de l'usine qu'il a entraperçu à l'occasion des stages. Nombreux sont ses camarades de classe qui ne souhaitent pas non plus poursuivre dans cette voie, à savoir le BTS CRSA : «après, on en a plein dans notre classe qui s'orientent [ailleurs]... ça ne leur plaît pas, ils voulaient juste un bac pour pouvoir passer... c'est juste ça, juste avoir le diplôme, le bac pour pouvoir aller dans une autre école après... » Cette défection collective à l'égard de la poursuite d'études «naturelle» de leur filière laisse les élèves de cette classe isolés, seuls face à la constitution de leurs vœux.

Le seul moyen dont Fabien semble disposer pour changer de voie est de calquer ses choix d'orientation sur ceux des élèves de terminale Électrotechnique énergie équipements communicants de son lycée, les «gars d'ELEEC » comme il les nomme, et qu'il fréquente en dehors des cours, à l'internat notamment. Fabien envisage en effet en premier vœu, et comme Anthony évoqué précédemment, la licence IMIS de l'université d'Angers, que beaucoup d'élèves en terminale professionnelle ELEEC de son lycée rejoignent, selon lui, chaque année. Fabien se raccroche en quelque sorte au wagon d'une orientation collective qui lui permet de fuir l'usine " [cette licence] déjà ce n'est plus dans le même secteur, ce n'est pas dans les usines, c'est totalement différent ", tout en étant assuré d'être pris : «et comme c'est l'université, de toute façon, c'est pris direct quand on a le bac...»

Cette pratique collective de l'orientation constitue ainsi un univers rassurant pour Fabien, lui permettant d'appréhender, à plusieurs, des démarches qui ne lui sont pas familières (se rendre au salon de l'apprentissage, au rendez-vous avec les responsables de la formation, rédiger sa lettre de motivation, etc.), et qui l'autorise ainsi à s'aventurer au-delà des frontières du BTS : 
Fabien : C'est des gars de l'ELEEC [...] qui m'ont parlé de la formation et tout et après je me suis dit... je me suis renseigné. Je crois que j'ai la fiche... [il cherche dans son sac]

Enquêtrice: Donc, tu en as parlé avec des élèves d'ELEEC et après tu es allé au salon, c'est ça?

Fabien : Je suis allé au salon avec des gars d'ELEEC...

Cette mobilisation collective, si elle permet à certains élèves de s'autoriser à choisir des parcours un peu singuliers, contribue néanmoins fortement à construire des destins communs plutôt limités, aussi bien dans l'espace abstrait de l'enseignement supérieur que dans l'espace géographique réel. Cette "solidarité au groupe» (Bernstein, 1975, p.49) contribue à renforcer la perception localisée de l'enseignement supérieur et freine fortement les projections au-delà des frontières du groupe de pairs. Les projets scolaires ne sont pas l'occasion de se distinguer des autres, mais au contraire d'envisager des projets conformes au groupe. Les bacheliers professionnels sont ainsi plus nombreux à penser que les études qu'ils envisagent « $n^{\prime} i m p o r t e ~ q u i$ pourrait les faire» $(38,5 \%)$, que les bacheliers technologiques $(30,3 \%)$ ou encore les bacheliers généraux $(22,7 \%)$. Au-delà d'une intériorisation de leurs positions respectives dans la hiérarchie des filières du secondaire, ces résultats traduisent aussi une moindre individualisation des projections scolaires chez les premiers.

L'encadrement par l'institution scolaire de la saisie des vœux dans l'application APB vient par ailleurs renforcer encore davantage cet effet de conservatisme scolaire ${ }^{18}$. En effet, alors que dans les classes de terminales générales la démarche est généralement opérée de manière individuelle par les élèves et à leur domicile, un grand nombre de lycéens professionnels rencontrés ont précisé que cette opération avait été effectuée dans une salle informatique de l'établissement, sous le contrôle d'un enseignant. L'internalisation de la procédure et l'organisation collective qui lui est liée participent ainsi également à minimiser l'expression des différences et des particularités individuelles (Bernstein, 1975) dans les choix opérés. Surtout, s'attacher aux conditions de l'expression des vœux dans APB permet de comprendre que la proximité ou la démarche collective, si elles constituent des valeurs propres aux élèves des terminales professionnelles, sont renforcées

18 Basil Bernstein parle du «conservatisme social» induit par I'usage du langage commun pour désigner une résistance à certaines formes de changements qui s'oppose à l'intérêt pour la nouveauté que favorise le langage formel (Bernstein, 1975, p.49). par l'institution scolaire. Ce sont ainsi les élèves les moins disposés à l'autonomie dans les choix d'études qui sont le plus assistés et les plus confortés dans un état de dépendance. L'influence des enseignants sur la poursuite d'études est ainsi plus importante pour les élèves de terminale professionnelle (dans le cadre d'une question fermée du questionnaire, 29,3\% d'entre eux les citent comme la plus forte «source de pression» dans le choix des études supérieures, contre $23,7 \%$ en terminale technologique et $20,3 \%$ en terminale générale). Mais avant même la procédure de candidature, la déclinaison différentielle des séances d'Accompagnement personnalisé (AP) selon les filières de baccalauréat en dit long sur la conception institutionnelle du choix en fonction du profil du candidat. Tandis que dans les classes de terminale générale et technologique enquêtées, les séances d'AP étaient l'occasion de la constitution d'un projet personnel (par exemple par la constitution d'un porte-folio individuel ou d'un web classeur qui archive, pour chaque élève, les sites internet et les ressources consultés pour l'orientation), pour les élèves de terminale professionnelle, ces séances étaient consacrées à des exercices collectifs de révision pour préparer la poursuite des études dans une formation ciblée, en l'occurrence le BTS attenant au baccalauréat.

\section{Conclusion}

"Si les enfants "butent" sur certaines difficultés, ce n'est pas à cause de leur manque $\mathrm{d}^{\prime \prime}$ "intelligence", mais plutôt parce que la nature de certains objets et des relations symboliques qui leur donnent leur sens (ici, les nombres et leurs implications) sont telles que nombre d'éléments pertinents ne sont pas perçus, et ne peuvent être retenus» (Bernstein, 1975, p. 53). À l'instar de l'analyse proposée par Basil Bernstein à propos des difficultés d'apprentissage du calcul des élèves de classes populaires, nous suggérons que les difficultés d'usage d'APB éprouvées par les élèves de terminale professionnelle et, plus largement, par les élèves des classes populaires, ne sont pas à interpréter comme le symptôme de "manques » mais plutôt comme l'expression d'un décalage entre deux façons de voir et de dire l'avenir scolaire et professionnel, chacune reposant sur une logique propre. L'application APB, loin de constituer un support neutre et transparent des choix des élèves, constitue un langage singulier de ces choix (ce que nous avons appelé la «logique formelle» de l'orientation) qui va favoriser ceux qui parlent la même langue (les bacheliers 
généraux et les bacheliers d'origine supérieure principalement) et qui va tenir à distance voire disqualifier les pratiques et les représentations de ceux qui arborent une autre logique, que nous avons appelée la «logique commune» de l'orientation.

\section{Bibliographie}

BEAUD S. (2002). $80 \%$ au bac et après... Les enfants de la démocratisation scolaire. Paris : La Découverte.

BEAUD S. \& PIALOUX M. (2001). «Les "bacs pro" à I'université. Récit d'une impasse ». Revue française de pédagogie, n ${ }^{136}$, p. 87-95.

BELORGEY N. (2010). L'hôpital sous pression. Enquête sur le «nouveau management public ». Paris : La Découverte.

BERNSTEIN B. (1975). Langage et classes sociales. Codes socio-linguistiques et contrôle social. Paris : Éd. de Minuit.

BERTHELOT J.-M. (1993). École, orientation, société. Paris : PUF.

BODIN R. \& ORANGE S. (2013). L'Université n'est pas en crise. Les transformations de l'enseignement supérieur : enjeux et idées reçues. Bellecombe-en-Bauges : Le Croquant.

BOURDIEU P. (1977). Algérie 60. Structures économiques et structures temporelles. Paris : Éd. de Minuit.

BOURDIEU P. \& BOLTANSKI L. (1975). «Le fétichisme de la langue». Actes de la recherche en sciences sociales, vol.1, $\mathrm{n}^{\circ} 4$, p. 2-32.

DANVERS F. (1988). «Pour une histoire de l'orientation professionnelle». Histoire de l'éducation, n³7, p. 3-15.

EYRAUD C. (2013). Le capitalisme au cœur de l'État. Comptabilité privée et action publique. Bellecombe-en-Bauges : Le Croquant.

ERLICH V. (1998). Les nouveaux étudiants. Un groupe social en mutation. Paris : Armand Colin.

FROUILLOU L. (2016). "Admission post-bac : un "libre choix" sous contrainte algorithmique. La production spatiale des inégalités par l'affectation informatique des étudiants du supérieur ». Justice spatiale, spatial justice, $\mathrm{n}^{\circ} 10$.

GOMBERT P. (2008). L'école et ses stratèges. Les pratiques éducatives des nouvelles classes supérieures. Rennes: Presses universitaires de Rennes.

HÉNOQUE M. \& LEGRAND A. (2004). L'évaluation de l'orientation à la fin du collège et au lycée. Rêves et réalités de l'orientation. Rapport au Haut Conseil de l'évaluation de l'école.

HENRIET A. \& PIETRYK G. (2012). Analyse de l'orientation et des poursuites d'études des lycéens à partir de la procédure admission post-bac. Rapport n²012-123, MEN

HETZEL P. (2006). De I'Université à l'emploi. Rapport au CNESER.

HOGGART R. ([1957] 1970). La culture du pauvre. Paris : Éd. de Minuit.
Claire Lemêtre

Université Paris 8-Vincennes-Saint-Denis, CIRCEFT-ESCOL

claire.lemetre@univ-paris8.fr

Sophie Orange

Université de Nantes, CENS sophie.orange@univ-nantes.fr

du mouvement d'orientation ». L'Orientation scolaire et professionnelle, $\mathrm{n}^{\circ} 1$, p. 3-43.

LEMÊTRE C. \& ORANGE S. (2017). «Le rapport à la certification des élèves de lycée professionnel : une mesure additionnelle des diplômes». Spirale, n59, p.41-51.

LUNEL P. (2007). «Pour un nouveau pacte avec la jeunesse». Schéma national de l'orientation et de l'insertion professionnelle, 27 mars 2007.

MENESR (2014). Repères et références statistiques sur les enseignements, la formation et la recherche, édition 2014. Paris : MENESR. En ligne : <http://cache.media.education.gouv. fr/file/2014/04/7/DEPP_RERS_2014_344047.pdf> (consulté le 8 juillet 2016).

ORANGE S. (2010). «Le choix du BTS. Entre construction et encadrement des aspirations des bacheliers d'origine populaire ». Actes de la recherche en sciences sociales, vol.3, $\mathrm{n}^{\circ} 183, \mathrm{p} .32-47$.

ORANGE S. (2013). L'autre enseignement supérieur. Les BTS et la gestion des aspirations scolaires. Paris : PUF.

PALHETA U. (2012). La domination scolaire. Sociologie de l'enseignement professionnel et de son public. Paris : PUF.

PISTOLESI N. (2015). «L'orientation active : une aide efficace pour choisir ses études? ». Regards croisés sur l'économie, vol. 1, n 16, p. 105-116.

REISS F. (2007). «Avis présenté au nom de la Commission des affaires culturelles, familiales et sociales sur le projet de loi de finances pour 2008 ( $n^{\circ} 189$ ). Tome IV. Enseignement scolaire». Avis n²77, Assemblée nationale.

ROCHEX J.-Y. (1995). «Interrogations sur le projet : la question du sens». Éducation permanente, $\mathrm{n}^{\circ} 125$.

SUAUD C. (1989). «Espace des sports, espace social et effets d'âge ». Actes de la recherche en sciences sociales, vol.79, $\mathrm{n}^{\circ} 1$, p. $2-20$.

THARIN I. (2005). Orientation, réussite scolaire: ensemble, relevons le défi. Rapport au Premier ministre.

TRUONG F. (2013). «La discipline du choix. De l'orientation scolaire après le bac en Seine-Saint-Denis ». Tracés, $\mathrm{n}^{\circ} 25$, p. $45-64$

VAN ZANTEN A. (2009). Choisir son école. Stratégies familiales et médiations locales. Paris : PUF.

VEZINAT N. (2012). Les métamorphoses de la Poste. Professionnalisation des conseillers financiers (1953-2010). Paris : PUF.

WILLIS P. (1978). «L'école des ouvriers». Actes de la recherche en sciences sociales, vol.24, $\mathrm{n}^{\circ} 1, \mathrm{p} .50-61$. 